\title{
China county-based prostate specific antigen screening for prostate cancer and a cost-effective analysis
}

\author{
Zhiguo Zhang ${ }^{1 \#, ~ G u o s h u ~ L i a n g ~}{ }^{2 \#}$, Peng Zhang ${ }^{3}$, Zhongqi Zhao ${ }^{4}$, Zhongnan $\mathrm{He}^{5}$, Fengzhen Luo ${ }^{6}$, \\ Zhenqing Chen ${ }^{7}$, Zongke Yang ${ }^{8}$, Zhijie Zhang ${ }^{9}$, Tao Xia ${ }^{10}$, Xin Liu ${ }^{11}$, Yong Zhang ${ }^{1}$, Wei Ye \\ ${ }^{1}$ Department of Urology, Liuyang People's Hospital, Liuyang, China; ${ }^{2}$ Department of Urology, Guanghan People's Hospital, Guanghan, \\ China; ${ }^{3}$ Department of Urology, Xinmi Hospital of T.C.M, Xinmi, China; ${ }^{4}$ Department of Urology, Pucheng County Hospital, Weinan, China; \\ ${ }^{5}$ Department of Urology, Ruichang People's Hospital, Ruichang, China; ${ }^{6}$ Department of Urology, The People's Hospital of Yudu County, Ganzhou, \\ China; ${ }^{7}$ Department of Urology, Xunxian People's Hospital, Hebi, China; ${ }^{8}$ Department of Urology, Dianjiang People's Hospital, Dianjiang, China; \\ ${ }^{9}$ Department of Urology, The Second Hospital of Qinhuangdao, Qinhuangdao, China; ${ }^{10}$ Department of Urology, Chengcheng County Hospital, \\ Weinan, China; ${ }^{11}$ Department of General Surgery, Linzhou People's Hospital, Linzhou, China \\ Contributions: (I) Conception and design: Y Zhang, W Ye; (II) Administrative support: Y Zhang, W Ye; (III) Provision of study materials or patients: Z \\ Zhang, G Liang, P Zhang, Z Zhao, Z He, F Luo, Z Chen, Z Yang, Z Zhang, T Xia, X Liu; (IV) Collection and assembly of data: Z Zhang, G Liang, \\ P Zhang, Z Zhao, Z He, F Luo, Z Chen, Z Yang, Z Zhang, T Xia, X Liu; (V) Data analysis and interpretation: Z Zhang, G Liang, Y Zhang, W Ye; (VI) \\ Manuscript writing: All authors; (VII) Final approval of manuscript: All authors. \\ "These authors contributed equally to this work and should be considered as co-first authors. \\ Correspondence to: Yong Zhang. Department of Urology, Liuyang People’s Hospital, Liuyang, China. Email: 2573495131@qq.com; Wei Ye. \\ Department of Urology, Guanghan People's Hospital, Guanghan, China. Email: ghyewei@163.com.
}

Background: Prostate cancer is one of the most common malignant tumors worldwide, and is the thirdleading cause of cancer death in men. Nearly $70 \%$ of new prostate cancer patients in China are locally advanced or widely metastatic with poor prognosis. Providing active treatment to early stage prostate cancer patients can improve the prognosis of prostate cancer patients. Thus, this study sought to evaluate the economy of early prostate specific antigen (PSA) screening for high-risk prostate cancer.

Methods: Based on the data collected from the PSA screening activities of 11 county hospitals from October 2019 to April 2021, this study evaluated a high-risk prostate cancer population who received PSA screening and their quality of life and economy. The screening population comprised males aged over 50 years. All screening patients were tested for PSA. If the PSA value is unnormal, a further diagnosis based on magnetic resonance imagining (MRI) or a transrectal ultrasound-guided prostate biopsy were performed. The decision-tree and Markov model was used to simulate the process of disease development of highrisk prostate cancer patients who underwent screenings and those who did not, and the incremental costeffectiveness ratio was also evaluated.

Results: A total of 13,726 men received a PSA screening. Of these, 1,062 men had abnormal PSA values, and 73 of these were diagnosed with prostate cancer. Of these 73 patients, 40, 21, and 12 had early stage, mid-stage, and late-stage prostate cancer, respectively. Compared to the patients unscreened, the 1,000 patients who received an early PSA screening increased their quality-adjusted life year (QALY) by 15.69 years; however, each QALY had an additional cost of 38,550 yuan, which was lower than the willingness to pay threshold of 72,447 yuan (per capita gross domestic product in 2020).

Conclusions: For high-risk prostate cancer patients, early screenings have a cost-effective advantage over no screenings. Thus, early screening should be vigorously promoted for high-risk prostate cancer patients.

Keywords: Prostate cancer; screening; cost-effectiveness analysis; China

Submitted Jul 16, 2021. Accepted for publication Oct 11, 2021.

doi: 10.21037/tau-21-779

View this article at: https://dx.doi.org/10.21037/tau-21-779 


\section{Introduction}

Prostate cancer is one of the most common malignant tumors worldwide, and is the third-leading cause of cancer death in men (1). The incidence of prostate cancer in men in China is lower than that in European and American countries; however, due to the acceleration of the aging population, and changes in lifestyle and diet, the incidence of prostate cancer in China has been increasing significantly in recent years. In 2009, the incidence of prostate cancer in China was 9.92 per 10 million (2). According to the 2015 Annual Report of Tumors in China, prostate cancer is 1 of the 6 cancers that increases the incidence rate in men, is ranked 9 in the top 10 malignant tumors, and is the 6th most common male malignancy (3). The study found that only $30 \%$ of new cases of prostate cancer in China were clinically localized at the time of diagnosis, and the remaining cases comprised patients with locally advanced or extensive metastasis who could not receive local curative treatment and had a poor prognosis (4). Thus, screening high-risk groups for prostate cancer, detecting patients with early stage prostate cancer and providing aggressive treatments are important ways to improve the prognosis of prostate cancer patients in China.

The purpose of tumor screening is to identify patients who have cancer in an apparently normal population. Earlier diagnosis and timely treatment can have a good curative effect and improve the prognosis of cancer patients. However, there are many controversies related to tumor screening, including those related to evaluations of the cost-effectiveness ratio of screening results, and social, ethical, and other issues (5-7). Given the late stage at which prostate cancer is initially diagnosed in China, the occult characteristics of early prostate cancer, the effective treatment for early prostate cancer, and the evidence of foreign research and literature reports, it is important to screen for prostate cancer in China.

There are many individually characterized methods for prostate cancer screening. Most experts recommend that the prostate specific antigen (PSA) test be used as the primary method of prostate cancer screening (8). In 2017, it was recommended that all male patients older than 50 years undergo PSA tests (9); however, controversy remains as to whether PSA tests are economical (10). For example, the European Randomized Study of Screening for Prostate Cancer (ERSPC) found that screening was not cost-effective based on a social willingness to pay (WTP) $\$ 100,000$ per life year gained (LYG) (7). A cost-effectiveness analysis of a prostate cancer screening program in Jinan, Shandong Province, China showed that while prostate cancer screening is associated with increased costs, it can detect prostate cancer earlier, increase the proportion of patients diagnosed with an early stage of the cancer, reduce metastatic cancer, reduce subsequent treatment costs, and is economically cost-effective [incremental cost-effectiveness ratio (ICER) = \$14,747/quality-adjusted life year (QALY)] (11).

To date, no study has conducted an economic evaluation of prostate cancer screening in China, and there is doubt as to whether prostate cancer screening based on PSA testing has any cost-effective advantages. In order to filled the data gap of prostate cancer PSA screening in rural China, this study sought to compare the cost-effectiveness of two intervention regimens of patients who received and patients who did not receive PSA screening to provide economic evidence of whether patients with high-risk prostate cancer should receive early PSA screenings. We present the following article in accordance with the CHEERS reporting checklist (available at https://dx.doi.org/10.21037/tau-21-779).

\section{Methods}

\section{Study population}

A total of 13,726 individuals at high risk of prostate cancer were screened for PSA from October 2019 to April 2021 at the Liuyang People's Hospital of Hunan Province, Guanghan People's Hospital of Sichuan Province, Xinmi Hospital of Traditional Chinese Medicine of Henan Province, Pucheng People's Hospital of Shanxi Province, Ruichang People's Hospital of Jiangxi Province, Yudu People's Hospital of Jiangxi Province, Xunxian People's Hospital of Henan Province, Dianjiang People's Hospital of Chongqing City, Qinhuangdao Second Affiliated Hospital of Liaoning Province, Chengcheng People's Hospital of Shanxi Province, and Linzhou People's Hospital of Henan Province.

This study was approved by the Ethics Committee of the Liuyang People's Hospital (approval number: 2021001), and all subjects signed the informed consent form. All procedures in this study involving human participants were conducted in accordance with the Declaration of Helsinki (as revised in 2013).

\section{Screening method}

The following screening process was adopted. All screening 
patients were tested for PSA. If the PSA was less than $4 \mathrm{ng} / \mathrm{mL}$, PSA screening every 2 years was recommended; however, if the PSA ranged from $4-10 \mathrm{ng} / \mathrm{mL}$ in patients aged 50 to 65 years or 4-20 ng/mL in patients aged $65-80$ years, a second PSA test was performed. If the results of the second PSA test still showed a PSA greater than $4 \mathrm{ng} / \mathrm{mL}$, a further diagnosis was made based on magnetic resonance imagining (MRI) or a transrectal ultrasound-guided prostate biopsy. Patients aged 50-65 years with a PSA of more than $10 \mathrm{ng} / \mathrm{mL}$ or patients aged 65-80 years with a PSA of more than $20 \mathrm{ng} / \mathrm{mL}$ underwent a MRI or a biopsy to confirm the diagnosis.

Data about the screening population was collected, including data about patients' height, weight, whether any immediate family members had suffered from prostate cancer, whether the patient had received a PSA test before, the latest PSA test value, whether the patient had received a prostate puncture within 3 years, whether the patient had taken prostate disease drugs previously, whether the patient had any chronic disease conditions, and whether the patient had other tumors or tumor types.

\section{Model design}

This study assessed the cost-effectiveness of screening tests for prostate cancer patients. The assessment was conducted from the payer's perspective. In this study, a decision-tree and Markov model was used to simulate the basic process of prostate cancer screening and the prognosis of prostate cancer patients. The costs and health benefits of being screened and not being screened were calculated using the model, and the cost-effectiveness of the two conditions were compared. The decision tree was partly used to simulate the screening process of prostate cancer. Under the tree, the screened patients could be allocated to three situations: no prostate cancer, prostate cancer found by screening, and prostate cancer with no screening. The three states were then used as the initial states to enter the Markov model for subsequent disease prognosis. The model structure is shown in Figure 1.

\section{Decision tree}

The decision-tree model assumed recurrent PSA screenings (with a time interval of 2 years) for 1,000 Chinese high-risk populations in each arm. These high-risk populations may or may not have prostate cancer. The detective rate was taken from a local PSA screening study.

(I) Individuals with PSA $<4 \mathrm{ng} / \mathrm{mL}$ should undergo PSA screening every 2 years;
(II) Individuals aged 50-65 years with a PSA of 4$10 \mathrm{ng} / \mathrm{mL}$ or individuals aged $65-80$ years with a PSA of 4-20 ng/mL should undergo a second PSA test. If the $2 \mathrm{nd}$ PSA results show a PSA of $<4 \mathrm{ng} / \mathrm{mL}$, the patients should undergo PSA screening every 2 years; if the 2 nd PSA results show a PSA of $>4 \mathrm{ng} / \mathrm{mL}$, the patients should be referred for further diagnosis by MRI or a transrectal ultrasonography (TRUS)-guided biopsy;

(III) Individuals aged 50-65 years with a PSA $>10 \mathrm{ng} / \mathrm{mL}$ or individuals aged $65-80$ years with PSA $>20 \mathrm{ng} / \mathrm{mL}$ should be referred for further diagnosis by MRI or a TRUS-guided biopsy.

After the screening and diagnosis process, high-risk patients were classified as "non-prostate cancer" patients and "screen-detected cancer" patients.

\section{Markov model}

The individuals in the decision-tree model then enter into the corresponding states in the Markov model. The nonprostate cancer individuals may remain in the same state or progress to a non-prostate cancer-specific death, or become screen-detected prostate cancer patients. Conversely, the screen-detected cancer patients may remain in the same state or progress to non-prostate cancer-specific death or prostate cancer-specific death.

Time horizon simulated in study was 20 years. A cycle was set as 1 year. The discount rate was set as $3.5 \%$ based on a recommendation of the China Guidelines for Pharmacoeconomic Evaluation (version 2019). China's per capita gross domestic (GDP) in 2020 of 72,447 yuan was set as the cost-effectiveness threshold (12). The costeffectiveness difference between being screened and not being screened was calculated, and the difference was then compared to the cost-effectiveness threshold to determine whether there were any cost-effective advantages related to being screened or not being screened.

The model parameters mainly comprised clinical efficacy parameters, cost parameters, and utility parameters. The clinical efficacy parameters included the proportion of patients screened and not screened in the high-risk prostate population, the proportion of patients diagnosed with nonprostate cancer and prostate cancer based on being screened and not being screened, the transition probability of 5 event nodes of non-prostate cancer patients, the prostate cancer patients identified by being screened, and the prostate cancer patients identified by not being screened, and death related to non-prostate cancer or death related 


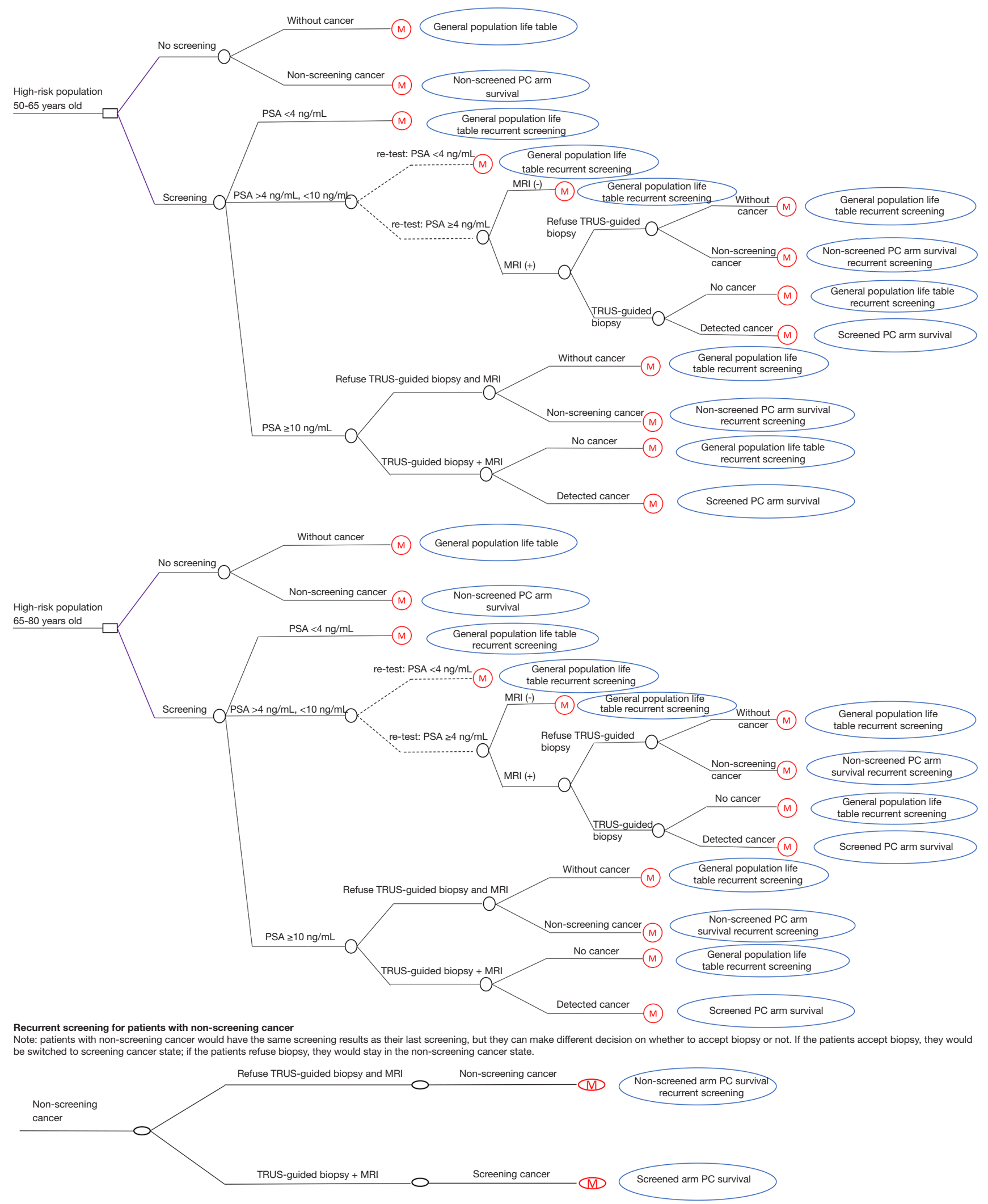

Figure 1 Model structure. 
to prostate cancer. The cost parameters included the costs of the above-mentioned event nodes, such as the costs of screening, prostate cancer treatment, and clinical care. In addition, the model also included utility parameters to characterize patients' quality of life. Among the clinical efficacy parameters, the proportion of patients who received screening or did not receive screening and the proportion of patients who were diagnosed "yes" or "no" for prostate cancer in the two intervention cases were calculated from the collected data in the model. The transition probability between the 5 event nodes in the Markov model was adopted from published literature (13). The utility parameters and the costs of the other event nodes were adopted from published literature (14), except for the screening costs, which were calculated using the collected data.

\section{Statistical analysis}

This study conducted a descriptive statistical analysis of quantitative data, including the number of cases, average number, standard deviation, minimum, maximum, median, and interquartile range (IQR). The qualitative data are presented as frequency and constituent ratios. $\mathrm{R}$ software was used to conduct the analysis. The economic model was run using Excel software. A one-way deterministic sensitivity analysis and probabilistic sensitivity analysis were performed to examine the effects of changes of different parameters on the modeling result. A one-way deterministic sensitivity analysis was conducted to change a certain model parameter or fix other parameters within a certain range, and then verify the influence of the numerical changes of the model parameters on the research results, which was usually represented by a tornado chart. In the sensitivity analysis, the transition probability, cost, and utility value of the model were increased and decreased by $20 \%$ on the basis of the baseline. A probabilistic sensitivity analysis was conducted to study the comprehensive effects of the variable parameters on the results of the Markov simulation by changing the variable influencing factors within a certain range to simulate the change degree of 1,000 random samples in Monte Carlo.

\section{Results}

\section{Screening results}

The PSA prostate cancer screening summary results are shown in Table 1. Of the 13,726 patients who underwent screening, 1,062 patients had abnormal PSA values (PSA $\geq 4$ ), $793(74.7 \%)$ patients had PSA values $\geq 4$ and $\leq 10$, and $269(25.3 \%)$ patients had PSA values $\geq 10$ (Table 2 ).

Of the 1,062 screened patients with abnormal PSA values, 709 (66.8\%) were reviewed. Of these, 483 (68.1\%) had PSA values $\geq 4$ and $\leq 10$, and $226(31.9 \%)$ had PSA values $\geq 10$. Further, $235(33.1 \%)$ of the reviewed patients were MRI positive. Of these MRI positive patients, 100 (20.7\%) had PSA values $\geq 4$ and $\leq 10$ and 135 (59.7\%) had PSA values $\geq 10$. A total of 224 (68.9\%) patients underwent a puncture, and of these, $48(48.5 \%)$ had PSA values $\geq 4$ and $\leq 10$, and $176(77.9 \%)$ had PSA values $\geq 10.73(32.6 \%)$ patients had positive paracentesis, and of these, 7 (14.6\%) had PSA values $\geq 4$ and $\leq 10$ and 66 (37.5\%) had PSA values $\geq 10$ (Table 3).

Of the 73 patients who confirmed with prostate cancer, $40(25.25 \%), 21(10.89 \%)$, and $12(9.41 \%)$ patients were diagnosed with early, mid- and late-stage prostate cancer (Table 4).

\section{Health economics assessment results}

The screening-related epidemiological data of patients were analyzed using an existing economic model (see Table 5 for the results). Compared to patients who did not receive an early screening, the 1,000 high-risk patients who received a screening had a QALY increase of 15.69 , and for each unit of incremental QALY, the treatment cost increased by 38,550 yuan. Setting a WTP of 1 times the GDP in 2020 (which according the Central People's Government of the People's Republic of China was 72,447 yuan), the increased cost of treatment was far lower than that of WTP, which indicates that compared to patients who did not receive a screening, patients who received an early screening had cost-effective advantages.

\section{Sensitivity analysis}

A one-way DSA and PSA were conducted to examine the effects of changes to different parameters on the modeling results. The results of the one-way DSA are shown in a tornado chart in Figure 2. Notably, the results showed that the utility of the screening attendance was the most influential factor. The results of the PSA are shown in Figure 3. Notably, the results showed that the probability of the cost-effective advantage of a screening was $100 \%$ when the model parameters were changed within the specified range. 
Table 1 Summary of the parameters included in the model

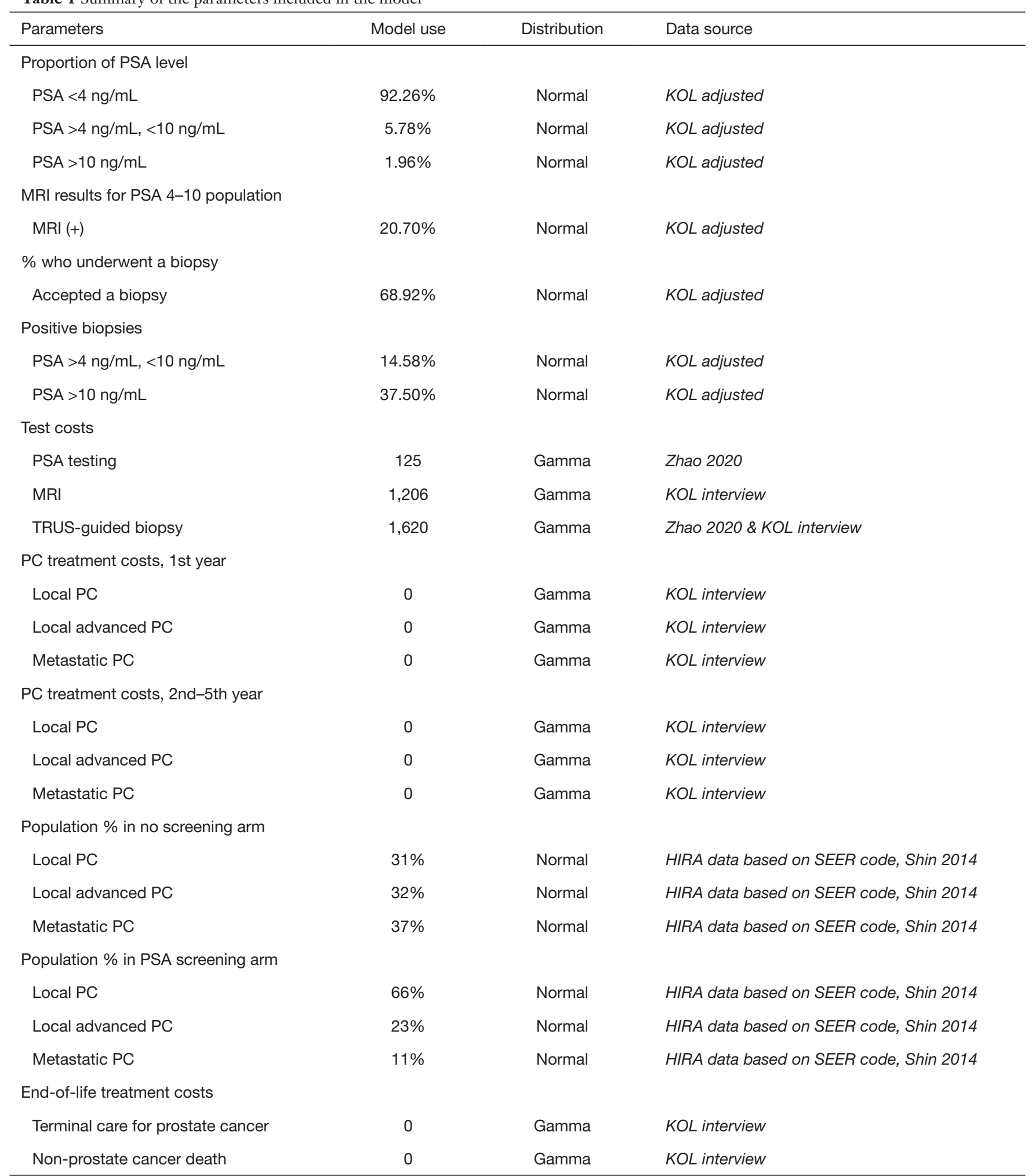

Table 1 (continued) 
Table 1 (continued)

\begin{tabular}{|c|c|c|c|}
\hline Parameters & Model use & Distribution & Data source \\
\hline Screening attendance & 0 & Beta & Heijnsdijk 2012 \\
\hline Biopsy & 0 & Beta & Heijnsdijk 2012 \\
\hline Local prostate cancer_1st year & 0 & Beta & Korean population, Shin 2014 \\
\hline Local advanced cancer_1st year & 0 & Beta & Korean population, Shin 2014 \\
\hline Local advanced cancer_2nd-5th year & 0 & Beta & Korean population, Shin 2014 \\
\hline Metastatic PC_1st year & 0 & Beta & Korean population, Shin 2014 \\
\hline Metastatic PC_2nd-5th year & 0 & Beta & Korean population, Shin 2014 \\
\hline \multicolumn{4}{|l|}{ Discount rate per cycle } \\
\hline Cost & $0.0 \%$ & Triangle & $\begin{array}{l}\text { China Guidelines for Pharmacoeconomic } \\
\text { Evaluation, version } 2019\end{array}$ \\
\hline Health outcome & $0.0 \%$ & Triangle & $\begin{array}{l}\text { China Guidelines for Pharmacoeconomic } \\
\text { Evaluation, version } 2019\end{array}$ \\
\hline
\end{tabular}

PSA, prostate specific antigen; MRI, magnetic resonance imaging; TRUS, transrectal ultrasonography; PC, prostate cancer.

Table 2 PSA prostate cancer screening summary

\begin{tabular}{lc}
\hline Indicator & Number of people (\%) \\
\hline Number of screening cases & 13,726 \\
PSA abnormal number & 1,062 \\
$4 \leq$ PSA $\leq 10$ & $793(74.7)$ \\
PSA $>10$ & $269(25.3)$ \\
\hline
\end{tabular}

Table 4 Summary of stages of patients diagnosed with prostate cancer

\begin{tabular}{lc}
\hline Diagnosis & Number $(\%)$ \\
\hline Early stage & $12(16.4)$ \\
Mid-stage & $21(28.8)$ \\
Late-stage & $40(54.8)$ \\
\hline
\end{tabular}

PSA, prostate specific antigen.

Table 3 PSA prostate cancer screening abnormal population examination summary

\begin{tabular}{lccc}
\hline Indicator & Population of abnormal, $\mathrm{n}(\%)$ & $4 \leq \mathrm{PSA} \leq 10, \mathrm{n}(\%)$ & $\mathrm{PSA}>10, \mathrm{n}(\%)$ \\
\hline Number of review (review rate) & $709(66.8)$ & $483(60.9)$ & $226(84.0)$ \\
Number positive (positive rate) & $235(33.1)$ & $100(20.7)$ & $135(59.7)$ \\
Real number of punctures (puncture rate) & $224(68.9)$ & $7(48.5)$ & $176(77.9)$ \\
Number of positive punctures (positive puncture rate) & $73(32.6)$ & $76(37.5)$ \\
\hline
\end{tabular}

PSA, prostate specific antigen.

\section{Discussion}

\section{Serious underdiagnosis of prostate cancer in rural China}

Prostate cancer is a malignant tumor, and is the third- leading cause of cancer death in men. In China, the incidence of prostate cancer is lower than that in European and American countries; however, the incidence of prostate cancer in China has been increasing year by year due to 
Table 5 Economic evaluation results

\begin{tabular}{lccc}
\hline & PSA screening & No screening & Difference \\
\hline Screening costs & $¥ 297,354$ & $¥ 0$ & $¥ 297,354$ \\
End-of life costs & $¥ 429,612$ & $¥ 448,669$ & $-¥ 19,057$ \\
PC-related treatment costs & $¥ 1,201,330$ & $¥ 874,751$ & $¥ 326,579$ \\
Total costs & $¥ 1,928,296$ & $¥ 1,323,420$ & $¥ 604,876$ \\
Total LY & $2,472.39$ & $2,446.09$ & 26.30 \\
Total QALY & $2,452.08$ & $2,436.39$ & 15.69 \\
ICER & - & - & $¥ 38,550 / \mathrm{QALY}$ \\
\hline
\end{tabular}

PSA, prostate specific antigen; PC, prostate cancer; LY, life year; QALY, quality-adjusted life year; ICER, incremental cost-effectiveness ratio.

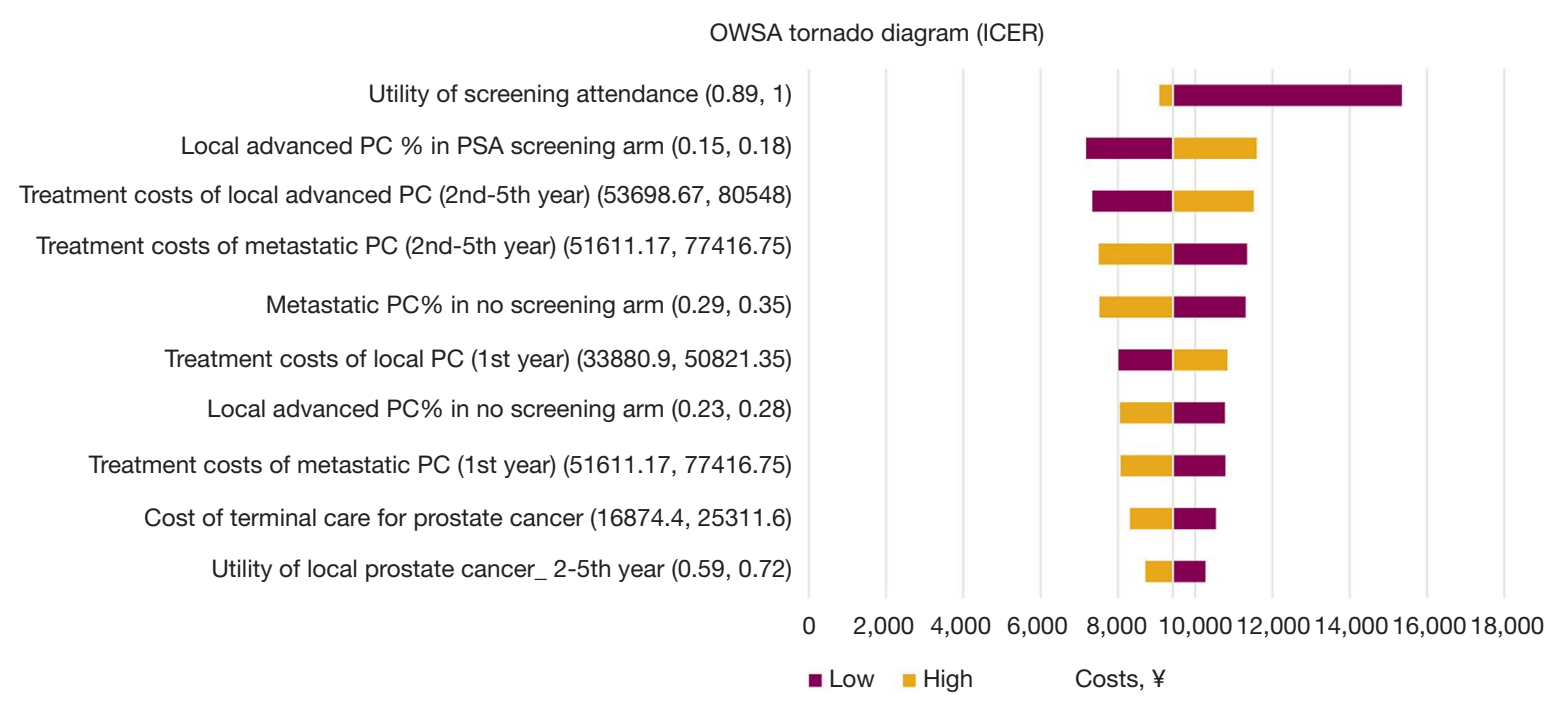

Figure 2 A 1-dimensional sensitivity analysis chart.

China's aging population and Westernized life habits (8). In more economically developed cities, the number of patients with prostate cancer has already surpassed the number of patients with bladder cancer and kidney cancer, and prostate cancer is now the top male genitourinary malignancy (3). A retrospective study based on cancer registry data of China's population showed that the 5 -year relative survival rate of men with prostate cancer in China from 2003 to 2015 was $66.4 \%$ (15), which is about the same as that of the United States (US) in 1975 (67.8\%). Notably, the 5-year relative survival rate in the US in 2009 was more than $98.6 \%$ (5).

The rural (county) population, which accounts for nearly half of China's population, is facing difficulties in the diagnosis and treatment of prostate cancer. According to the China Cancer Registry Annual Report of 2018 (16), the incidence of prostate cancer in the city is 8.10 per 100,000 (ASR World), and the incidence in the county is 3.97 per 100,000 (ASR World). Given that the risk factors of prostate cancer (e.g., age, race, heredity factors, and a diet high in animal fats) are similar between the city and county in China, there number of patients with prostate cancer in rural populations may be seriously underdiagnosed.

\section{Prostate cancer PSA screening promotes the early diagnosis and treatment of rural patients}

In recent years, many prostate cancer molecular biomarkers and imaging methods have been used to conduct auxiliary 


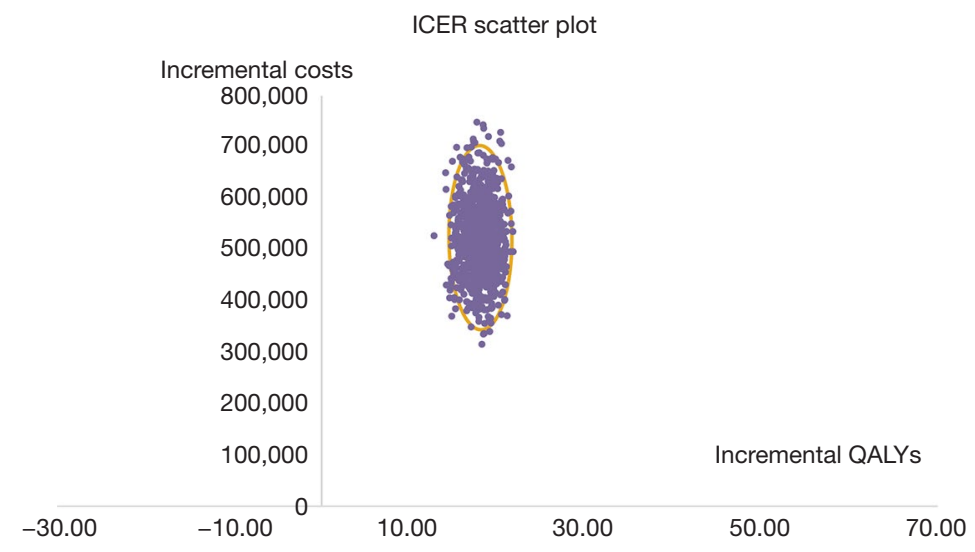

Figure 3 A one-way probability sensitivity analysis chart.

examinations of prostate cancer; however, the classic PSA detection test is still recommended and relied on in the current guidelines and used in clinical practice to screen prostate cancer. PSA screening has a number of advantages, including its simplicity, low costs, and relative accuracy. PSA screening can effectively screen out a large number of patients at high risk of prostate cancer, and can further lead to the early diagnosis and treatment of prostate cancer patients, which in turn improves the treatment effects and prognosis of patients with prostate cancer.

The screening of prostate cancer based on PSA has been controversial historically. It can improve the detection rate of patients with early prostate cancer, for whom timely intervention treatments can be provided to reduce cancer mortality, improving the number of patients who survive; however, the accuracy of PSA screening in non-specific populations is low, and PSA screening can easily lead to unnecessary puncture biopsies or even treatments, resulting in increased treatment costs, side effects, complications, and anxiety.

The two most controversial studies are the prostate, lung, colorectal, and ovarian (PLCO) study in the US, and the European randomized study of screening for prostate cancer (ERSPC) in Europe. Opposite conclusions were drawn from the results of these two studies. The ERSPC results indicated that PSA screening reduced mortality by $27.0 \%$ and metastatic prostate cancer by $48.9 \%$ (17). Conversely, the PLCO results indicated that there was no significant difference in prostate cancer-related mortality between screened and non-screened patients (6). However, some new studies have indicated that the reliability of the results is greatly reduced due to methodological flaws in the PLCO tests, including a high contamination rate (more than $70 \%$ of the population in the control group also underwent PSA examination during the study period). Some later studies reanalyzed the data of the PLCO study and drew the same conclusion as that of the ERSPC study; that is, that screening can reduce prostate cancer-related mortality $(1,7)$.

The development history of PSA screening shows that PSA screening can have direct or indirect effects on the incidence and mortality of prostate cancer. In the US, PSA screening has caused the incidence of prostate cancer to fluctuate greatly, and the mortality rate to change. In the early 1990s, PSA screening was approved by the Food and Drug Administration in the United States, and was widely used in unselected men. The results showed that the detection rate of prostate cancer increased sharply. However, around 1995, a number of reports stated that prostate cancer was found in the autopsy results of elderly men who had died of other diseases. Many prostate cancer patients who were diagnosed after tests and returned abnormal PSA values survived for 15 to 20 years, and eventually died of other diseases (1). Such results increased the controversies and challenges surrounding PSA screenings. In 2008, the US Preventive Services Task Force (USPSTF) recommended PSA detection downgraded to be $\mathrm{D}$ in all men so as not to over diagnose and over treat, and the incidence of prostate cancer then dropped sharply. The prevalence of PSA detection remained stable from 2013 to 2015 . However, some later reports indicated that the incidence of invasive prostate cancer has been increasing. For example, an analysis based on cancer registration data covering $89 \%$ of the US population showed that the overall decline in the incidence of prostate cancer had been concealing increases in the number of long-term diagnoses 
in different age groups and races since 2010. In 2017, the USPSTF revised its recommendations on PSA screening for men aged 55-69 years, adjusting the recommended grade to $\mathrm{C}$, and stated that "in some men, screening has potential benefits in reducing prostate cancer mortality." At present, the major international guidelines for prostate cancer PSA screening still retain a positive attitude, but the application method is slightly different.

While prostate cancer screening remains controversial, data suggest an improvement in prostate cancer mortality and increased detection of metastatic disease. Continued prostate cancer screening in the appropriate patients can prevent aggressive prostate cancers, metastatic disease, and reduce mortality. Thus, PSA could be used with other screening tools to better select patients for further evaluation with prostate biopsy (18).

The latest Chinese prostate cancer diagnosis and treatment standards, prostate cancer screening expert consensus, and foreign-related guidelines all indicate that prostate cancer screening is of great value to high-risk groups $(9,19,20)$. The current definition of high-risk groups is men aged over 50 years, or men aged over 45 years with a family history of prostate cancer, or men aged over 40 years with a high PSA value. In the past, prostate cancer screening programs based on PSA have been carried out in many cities in China. For example, from 1998 to 2000, 4,218 men aged over 50 years were screened for PSA in Changchun, and 29 patients with prostate cancer were diagnosed (after adjusting for age, the detection rate for prostate cancer was $1.28 \%$ ) (21). From 2004 to 2005, 8,562 men aged over 50 years were screened for PSA in Nanjing, and 58 patients with prostate cancer were diagnosed (the detection rate was $0.68 \%)(22)$.

Some studies took a healthcare perspective for the prostate cancer screening, including the cost of procedures, equipment and resource use (staff time) associated with the screening test, biopsy, treatments, general practitioner visits, hospital admissions and procedures, as well as terminal care costs (23-25). A US study, which showed prostate cancer screening was cost-effective, took a US payer perspective, where healthcare perspective costs were included, but the inclusion of out of pocket costs to patients was unclear (26).

There is no report on prostate cancer screening in rural China. In the present study, 13,726 high-risk prostate cancer patients were screened in 11 health service centers in rural China. The abnormal rate of PSA was $7.74 \%$, which was similar to that in Nanjing and Changchun. Among the screened patients, 224 patients received a biopsy, and
$73(0.53 \%)$ patients were diagnosed with prostate cancer. Among the detected patients, $65.75 \%$ were in the early stage, $23.29 \%$ were in the local progressive stage, and $10.96 \%$ were in the late stage. The proportion of metastasis was only $34.25 \%$, which was much lower than the $69 \%$ of the non-screening population. Our results fill gaps about prostate cancer screening in rural China.

\section{Prostate cancer screening in rural China is cost effective, but there is still significant room for improvement}

The cost-effectiveness analysis of a prostate cancer screening project in Jinan, Shandong Province, China showed that while prostate cancer screening has increased costs compared to no screening, it detects patients earlier, increases the probability of finding patients with early cancer, reduces metastatic cancer, and reduces follow-up treatment costs. Thus, prostate cancer screening is cost effective from an economical perspective (ICER $=14,747$ yuan/QALY) (11). Based on the screening scheme adopted in the study, the ICER of PSA screening compared to that of non-screening was 38,550 yuan/QALY, is way less than the standard of 3 times of per capita GDP, WTP mentioned by the World Health Organization and widely referenced in academic circles of 194,169 yuan. Thus, carrying out PSA screenings in rural China has a cost-effectiveness advantage.

However, there was a great gap between the results of this study (of 38,550 yuan/QALY) and the results of a study of PSA screening in an urban population (of 14,747 yuan/QALY). Comparing both screening data, the gap is mainly due to the:

(I) Low review rate: In the present study, only $66.8 \%$ of patients with abnormal PSA values underwent further review, which resulted in relatively higher screening costs. Conversely, in the urban screening, the review rate reached $98 \%$. The main reason for this difference is that the compliance of the rural population was relatively poor, compared with urban population;

(II) Low puncture acceptance rate: In the review population, only $68.4 \%$ of suspected patients with puncture indications received a puncture biopsy. Notably, the proportion of patients with a PSA value of $4-10 \mathrm{ng} / \mathrm{dL}$ was as low as $48.5 \%$ (the proportion of patients in the urban population was $68.9 \%)$, which not only increased the screening costs, but also greatly affected the staging distribution of the detected patients. In this study, we showed that doctors in county hospitals were 
more likely to recommend a puncture biopsy to suspected patients with higher PSA values, which is closely related to the lower positive rate of punctures in patients with lower PSA values;

(III) Low positive rate of puncture: For patients with PSA values of $4-10 \mathrm{ng} / \mathrm{dL}$ and PSA values $>10 \mathrm{ng} / \mathrm{dL}$, the positive rates of puncture in this study were $14.58 \%$ and $37.50 \%$ respectively (compared to $21 \%$ and $39 \%$ in cities), and the positive rate of PSA values ranging from $4-10 \mathrm{ng} / \mathrm{dL}$ was low. The study showed that the positive rate of puncture was directly proportional to the PSA data. In this study, the positive rate of the group of patients with PSA values $>10$ was higher than that of patients with PSA values of $4-10 \mathrm{ng} / \mathrm{dL}$, which is in line with objective reality. However, the positive rate of the group of patients with PSA values of $4-10 \mathrm{ng} / \mathrm{dL}$ in rural areas was lower than that in urban areas. The differences between the urban and rural areas may be related to the availability of puncture equipment and the technical competency levels of staff. The afore-mentioned facts aggravated the tendency of doctors to administer punctures to patients with higher PSA values in county hospitals;

(IV) Late staging: In this study, 65.75\%, 23.29\%, and $10.96 \%$ of the patients were in early stage, locally advanced stage, and late stage, respectively, compared to $81.72 \%, 16.13 \%$, and $2.15 \%$, respectively, of patients in urban areas. The proportion of patients with metastatic cancer (including lymph node metastasis and distant metastasis) in rural areas was much higher than that in urban areas $(34.25 \%$ vs. $14.28 \%)$. The higher treatment costs and lower survival benefits derived by metastatic patients directly led to the ICER value of this study greatly exceeding the cost-effectiveness evaluation results for urban screenings. Previous studies have indicated that the level of PSA serum of prostate cancer patients in the late stage is significantly higher than that of prostate cancer patients in the early stage. The tendency of the patients in urban area having higher PSA receiving more punctures was the reason why many patients were not detected to have prostate cancer until they were in the late stage.

Above all, it is urgent to improve the level of puncture equipment and technology available in county hospitals in China, and improve the concept of determining whether puncture is needed based on PSA values. Additionally, follow-up examinations of screened abnormal populations are needed to further improve the incremental costeffectiveness ratio of PSA screenings for prostate cancer.

\section{Prostate cancer screening in rural areas in China can reduce personal and socioeconomic burdens}

Thirty years ago, when digital rectal examination was the only diagnostic method, $30-35 \%$ and $45-50 \%$ of patients initially diagnosed with prostate cancer in the US had bone metastasis and lymph node metastasis, respectively, which is very similar to the current situation in China. Staging and metastasis are the most important prognostic factors for prostate cancer. The 5 -year survival rate of metastatic patients is less than $30 \%$, while that of localized prostate cancer is close to $100 \%$. It can be inferred that only $4-5 \%$ of new prostate cancer patients in the US are metastatic as a result of PSA screening. Due to PSA screening, the median age of death in the US from 2012 to 2016 was as high as 80 years old. At present, due to the late diagnosis in rural China, the proportion of prostate metastatic tumors (including lymph node metastasis and distant metastasis) is $69 \%$, which is much higher than that of $57 \%$ for urban patients. Additionally, in the poor treatment condition, the 5 -year relative survival rate of rural patients with prostate cancer was much lower than that of urban patients (62.4\% vs. $70.8 \%)$. Studies have shown that the prognosis of metastatic prostate cancer is very poor $(27,28)$. Using existing treatment methods, localized prostate cancer can be cured by radical treatment. However, after tumor metastasis, the life of patients can only be prolonged using long-term drug-based treatments, which place heavy burdens on rural patients, their families and society.

In this study, patients in the early, locally advanced, and late stages comprised $65.75 \%, 23.29 \%$, and $10.96 \%$ of prostate cancer patients, respectively. The proportion of metastasis was only $34.25 \%$, which is a figure far lower than the $69 \%$ of the non-screening population, which greatly improved the survival time of patients and reduced the personal and social economic burdens.

In conclusion, the diagnosis and treatment of prostate cancer in rural China has a long way to go. This study filled the data gap of prostate cancer PSA screening in rural China by conducting a data analysis of multi-center screenings and a cost-effectiveness evaluation. This study also analyzed the cost-effectiveness of prostate cancer screening from the perspective of health economics, and quantified the direct and indirect benefits of prostate cancer screening, 
and showed that prostate cancer screening can reduce labor losses and generate indirect benefits. The results of this study provide scientific evidence for government decisionmaking agencies to develop regional prostate cancer screening programs. If more data from prostate cancer screening projects are collected in the future, the findings could be updated using the health economics model constructed in this study, and more real-world data could be used to evaluate prostate cancer screening across different regions and populations, and in relation to different strategies.

\section{Conclusions}

For high-risk prostate cancer patients, receiving an early screening has a cost-effective advantage over no screening. Thus, early screening should be vigorously promoted to high-risk prostate cancer patients.

\section{Acknowledgments}

Funding: None.

\section{Footnote}

Reporting Checklist: The authors have completed the CHEERS reporting checklist. Available at https://dx.doi. org/10.21037/tau-21-779

Data Sharing Statement: Available at https://dx.doi. org/10.21037/tau-21-779

Conflicts of Interest: All authors have completed the ICMJE uniform disclosure form (available at https://dx.doi. org/10.21037/tau-21-779). The authors have no conflicts of interest to declare.

Ethical Statement: The authors are accountable for all aspects of the work, including ensuring that questions related to the accuracy or integrity of any part of the work have been appropriately investigated and resolved. This study was approved by the Ethics Committee of the Liuyang People's Hospital (approval number: 2021-001), and all subjects signed the informed consent form. All procedures in this study involving human participants were conducted in accordance with the Declaration of Helsinki (as revised in 2013).

Open Access Statement: This is an Open Access article distributed in accordance with the Creative Commons Attribution-NonCommercial-NoDerivs 4.0 International License (CC BY-NC-ND 4.0), which permits the noncommercial replication and distribution of the article with the strict proviso that no changes or edits are made and the original work is properly cited (including links to both the formal publication through the relevant DOI and the license). See: https://creativecommons.org/ licenses/by-nc-nd/4.0/.

\section{References}

1. Catalona WJ. Prostate Cancer Screening. Med Clin North Am 2018;102:199-214.

2. Liu S, Cai H, Cheng W, et al. Association of VDR polymorphisms (Taq I and Bsm I) with prostate cancer: a new meta-analysis. J Int Med Res 2017;45:3-10.

3. Zheng RS, Sun KX, Zhang SW, et al. Report of cancer epidemiology in China, 2015. Zhonghua Zhong Liu Za Zhi 2019;41:19-28.

4. Yu W, Zhou L. Early Diagnosis of Prostate Cancer from the Perspective of Chinese Physicians. J Cancer 2020;11:3264-73.

5. Schröder FH, Hugosson J, Roobol MJ, et al. Screening and prostate cancer mortality: results of the European Randomised Study of Screening for Prostate Cancer (ERSPC) at 13 years of follow-up. Lancet 2014;384:2027-35.

6. Shoag JE, Mittal S, Hu JC. Reevaluating PSA Testing Rates in the PLCO Trial. N Engl J Med 2016;374:1795-6.

7. Tsodikov A, Gulati R, Heijnsdijk EAM, et al. Reconciling the Effects of Screening on Prostate Cancer Mortality in the ERSPC and PLCO Trials. Ann Intern Med 2017;167:449-55.

8. Wang D, Li P, Ji Z, et al. Review and summary of health screening and early diagnosis and treatment of prostate cancer in China. Int J Surg 2020;47:577-81.

9. Wang Y, Dai B. Explanation of Consensus of prostate cancer screening, version 2017. Journal of Clinical Surgery 2018;26:15-8.

10. Sanghera S, Coast J, Martin RM, et al. Cost-effectiveness of prostate cancer screening: a systematic review of decision-analytical models. BMC Cancer 2018;18:84.

11. Zhao G, Shao Y, Zhang N, et al. Cost-Effectiveness Analysis of Prostate-Specific Antigen Screening Among Chinese Men. Value Health Reg Issues 2020;21:272-9.

12. National Bureau of Statistics, Statistical communique of the people's republic of China on the 2019 national 
economic and social development, China Statistics Press. Available online: http://www.stats.gov.cn/tjsj/zxfb/202102/ t20210227_1814154.html (access date : 17th May 2021).

13. Shin S, Kim YH, Hwang JS, et al. Economic evaluation of prostate cancer screening test as a national cancer screening program in South Korea. Asian Pac J Cancer Prev 2014;15:3383-9.

14. Heijnsdijk EA, Wever EM, Auvinen A, et al. Quality-oflife effects of prostate-specific antigen screening. N Engl J Med 2012;367:595-605.

15. Zeng H, Chen W, Zheng R, et al. Changing cancer survival in China during 2003-15: a pooled analysis of 17 population-based cancer registries. Lancet Glob Health 2018;6:e555-67.

16. National Cancer Center. 2018 China Cancer Registry Annual Report. People's Medical Publishing House, 2018.

17. Schröder FH, Hugosson J, Roobol MJ, et al. Screening and prostate-cancer mortality in a randomized European study. N Engl J Med 2009;360:1320-8.

18. Robin TP, Geiger CL, Callihan EB, et al. Prostate Cancer in Older Adults: Risk of Clinically Meaningful Disease, the Role of Screening and Special Considerations. Curr Oncol Rep 2021;23:130.

19. Howard K, White S, Salkeld G, et al. Cost-effectiveness of screening and optimal management for diabetes, hypertension, and chronic kidney disease: a modeled analysis. Value Health 2010;13:196-208.

20. Han Y, Yan T, Ma H, et al. Cost-Effectiveness Analysis of Helicobacter pylori Eradication Therapy for Prevention of Gastric Cancer: A Markov Model. Dig Dis Sci 2020;65:1679-88.

Cite this article as: Zhang Z, Liang G, Zhang $P$, Zhao Z, He Z, Luo F, Chen Z, Yang Z, Zhang Z, Xia T, Liu X, Zhang Y, Ye W. China county-based prostate specific antigen screening for prostate cancer and a cost-effective analysis. Transl Androl Urol 2021;10(10):3787-3799. doi: 10.21037/tau-21-779
21. Li X, Tsuji I, Kuwahara M, et al. Mass screening of prostate cancer in Changchun City of China. Int Urol Nephrol 2004;36:541-8.

22. Hua L, Qiao D, Xu B, et al. Clinical and pathological characteristics of screen-detected versus clinically diagnosed prostate cancer in Nanjing, China. Med Oncol 2011;28:357-64.

23. Heijnsdijk EA, de Carvalho TM, Auvinen A, et al. Costeffectiveness of prostate cancer screening: a simulation study based on ERSPC data. J Natl Cancer Inst 2015;107:366.

24. Pataky R, Gulati R, Etzioni R, et al. Is prostate cancer screening cost-effective? A microsimulation model of prostate-specific antigen-based screening for British Columbia, Canada. Int J Cancer 2014;135:939-47.

25. Keller A, Gericke C, Whitty JA, et al. A Cost-Utility Analysis of Prostate Cancer Screening in Australia. Appl Health Econ Health Policy 2017;15:95-111.

26. Roth JA, Gulati R, Gore JL, et al. Economic Analysis of Prostate-Specific Antigen Screening and Selective Treatment Strategies. JAMA Oncol 2016;2:890-8.

27. Qi JL, Wang LJ, Zhou MG, et al. Disease burden of prostate cancer among men in China, from 1990 to 2013. Zhonghua Liu Xing Bing Xue Za Zhi 2016;37:778-82.

28. National Health Commission of the People's Republic of China. Chinese guidelines for diagnosis and treatment of prostate cancer 2018. Available online: http://www.nhc. gov.cn/yzygj/s7659/201812/b21802b199814ab7b1219b87 de0cae51.shtml (accessed date: 11th August 2021).

(English Language Editor: L. Huleatt) 